\title{
FORMATION OF SINGLE CHARGED DROPS IN LIQUID MEDIA UNDER A UNIFORM ELECTRIC FIELD
}

\author{
TORU TAKAMATSU, MANABU YAMAGUCHI AND TAKASHI KATAYAMA \\ Department of Chemical Engineering, Faculty of Engineering Science, \\ Osaka University, Toyonaka, Osaka 560
}

\begin{abstract}
Volumes and charges of water drops formed in a uniform electric field $(0-4 \mathrm{kV} / \mathrm{cm})$ have been measured in cyclohexane, toluene, insulating oil and three kinds of silicone oils as continuous phase. Effects of nozzle length extended from an electrode, flow rate of dispersed phase and liquid viscosity of continuous phase on the volume and the charge are examined. Experimental values are compared with those calculated by equations presented.

It is clarified that experimental volumes of the drops formed in a liquid having viscosity up to ten centipoises can be predicted by the presented equation. The experimental drop charges at low flow rates of dispersed phase are also represented by the equation. In a range of high flow rates of dispersed phase, however, a sudden decrease of charge has been observed.
\end{abstract}

\section{Introduction}

The formation of electrically charged drops in an electric field has been studied by several investigators ${ }^{5,7,8,15)}$. Most interest, however, has been focused on the atomization of electrified liquids in the atmosphere, because the charged drops are used mainly in gaseous phase, as in the case of spray painting.

For liquid-liquid system, Stewart and Thornton ${ }^{12 \text { ? }}$ proposed the application of an electric field as a new technique to increase the rate of mass transfer in liquidliquid extraction and studied the formation of charged water-drops in $n$-hexane under an applied electric field. Afterward some investigator ${ }^{1,4,14)}$ reported significant enhancement of mass transfer rates between dispersed and continuous phases by applying electric fields. However, no detailed investigation has been made of charged drop formation in liquid media.

In our previous study ${ }^{13}$, theoretical equations were derived for the volume and the charge of electrically conductive liquid drops formed in a uniform electric field and experiments were made in the atmosphere. It was found possible to represent the experimental data satisfactorily by the theoretical equations.

The present paper deals with formation of charged drops in liquid media in a uniform electric field as a fundamental study of "electrostatic liquid-liquid extraction". The volumes and the charges of single water drops formed in the uniform electric field were measured over a wide range of dispersed-phase flow

Received February 5, 1982. Correspondence concerning this article should be addressed to T. Katayama. rates with several dielectric liquids as the continuous phase. A theoretical equation for predicting the charged drop volume was also developed by taking account of electrostatic force in an equation of the Scheele and Meister type.

\section{Theoretical}

Some investigators ${ }^{6,8,11)}$ have reported studies of uncharged drop formation at a single nozzle tip submerged in a liquid phase. They predicted the drop volume by assuming the formation process to be divided into two stages: In the first stage, a pendant drop expands until the force balance acting on the drop at the nozzle tip breaks down. The force balance is composed of gravitational and inertial forces as drawing ones and viscous and interfacial tension forces as restraining ones. In the second stage, when the drawing force exceeds the restraining force, the drop begins to break away from the nozzle, and the drop continues its necking process. Scheele and Meister ${ }^{11}$ proposed the following equation for predicting the drop volume by using the two-stage drop formation model.

$$
\begin{aligned}
\phi v= & \frac{\pi D_{\mathrm{n}} \sigma}{\Delta \rho g}+\frac{20 \mu_{\mathrm{c}} Q_{\mathrm{n}} D_{\mathrm{n}}}{d_{\mathrm{e}}^{2} \Delta \rho g}-\frac{4 \rho_{\mathrm{d}} Q_{\mathrm{n}} u_{\mathrm{n}}}{3 \Delta \rho g} \\
& +K\left(\frac{Q_{\mathrm{n}}^{2} D_{\mathrm{n}, \mathrm{in}}^{2} \rho_{\mathrm{d}} \sigma}{(\Delta \rho g)^{2}}\right)^{1 / 3}
\end{aligned}
$$

where the Harkins factor was taken into account and $K$-value in the equation was evaluated to be 4.5 for twelve liquid-liquid systems used in their experiments. 


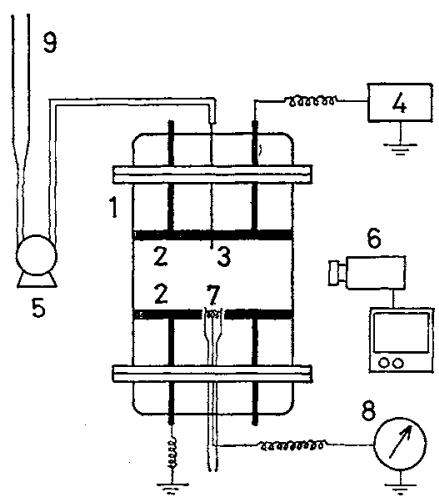

$\begin{array}{llll}1 & \text { Glass column } & 6 & \text { TV system } \\ 2 & \text { Copper plate } & 7 & \text { Platinum net } \\ 3 & \text { Nozzle } & 8 & \text { Electrometer } \\ 4 & \text { H. V. generator } & 9 & \text { Burette } \\ 5 & \text { Micro-tube pump } & & \end{array}$

Fig. 1 Schematic diagram of experimental apparatus

If the formation process of an electrically charged drop is the same as that of an uncharged drop, electrostatic force $\left(F_{\mathrm{e}}\right)$ must be added to the foregoing force balance as a drawing force.

$$
\begin{array}{r}
\phi \Delta \rho g v-20 \mu_{\mathrm{c}} Q_{\mathrm{n}} D_{\mathrm{n}}\left(\frac{\pi}{6}\right)^{2 / 3} v^{-2 / 3}+F_{\mathrm{e}}-\pi D_{\mathrm{n}} \sigma \\
+\frac{4}{3} \rho_{\mathrm{d}} Q_{\mathrm{n}} u_{\mathrm{n}}-K\left(Q_{\mathrm{n}}^{2} D_{\mathrm{n}, \mathrm{n}}^{2} \rho_{\mathrm{d}} \sigma \Delta \rho g\right)^{1 / 3}=0
\end{array}
$$

In the previous study ${ }^{13)}$, the force acting and the charge induced on a pendant drop in a uniform electric field were derived as Eqs. (3) and (4) by assuming that the drop is a spherical conductor and that the charge induced on the nozzle extended from an electrode is negligible.

$$
\begin{aligned}
F_{\mathrm{e}} & =4 \pi \varepsilon \beta r^{2} E_{0}^{2} \\
Q & =4 \pi \varepsilon \gamma r^{2} E_{0}
\end{aligned}
$$

where $\beta$ and $\gamma$, respectively, are the force and charge coefficients which were given analytically. The values could be approximated as follows:

$$
\begin{aligned}
& \beta= \begin{cases}1.12 & (\alpha=1) \\
\alpha+0.34 & (\alpha>1)\end{cases} \\
& \gamma=\alpha+0.63 \quad(\alpha \geq 1)
\end{aligned}
$$

where

$$
\alpha=(l+r) / r
$$

By solving Eq. (2) with Eq. (3) we obtain charged drop volumes at each electric field strength (=applied potential/electrode gap) for a given flow rate of dispersed phase. The electric charge on the detached drop is assumed to be equal to that on the pendant drop even though the volumes are different, because most of the charge accumulates on the front surface of the drop which detaches from the nozzle.

\section{Experimental}

A schematic diagram of the experimental apparatus is shown in Fig. 1. A glass column (1) equipped with a pair of electrodes was filled with a dielectric liquid as the continuous phase. Upper and lower electrodes (2), made of copper disks, had a diameter of $11 \mathrm{~cm}$, the same size as the inner diameter of the column, and were kept $5 \mathrm{~cm}$ apart. This arrangement of the electrodes was determined so as to obtain a uniform electric field ${ }^{13)}$. A stainless steel nozzle $(0.1 \mathrm{~cm}$ O.D., $0.06 \mathrm{~cm}$ I.D.) (3) was extended from the center of the upper electrode and connected, together with the electrode, to a high-voltage generator (Brandenburg 2807R) (4). The applied voltage for the experiments was varied from 0 to $20 \mathrm{kV}$ (positive polarity) and the lower electrode was earthed. No influence of polarity on drop volume and charge was found in the present study.

The drop volume was calculated from drop formation time and dispersed-phase flow rate controlled by a micro-tube pump (5). When the frequency of drop formation was high, forming behavior was recorded on a TV system (6) and formation time was measured by reproducing the pictures at a low speed. The charged drop fell through the continuous liquid phase and contacted a platinum net (7), which was insulated from the rest of the lower earthed electrode. The drop charge was measured by an electrometer (Takeda Riken TR8651) (8) connected to the net. The electrometer has a current compensation function which offsets the false current. Net charge of a drop could be measured by eliminating the current flowing through the continuous phase by setting the current compensator for each run. Experiments were made to examine the effects of the following three factors on the volume of a charged drop and charged characteristic of the drop. 1) The effect of liquid viscosity of continuous phase: the dielectric liquids used as the continuous phase were cyclohexane, toluene, insulating oil (Nisseki A) and three kinds of silicone oils (Shin-etsu KF96L(1.0), KF96(10) and KF96(100), abbreviated as silicone oil-A, oil-B, and oil-C, respectively, in this paper). The continuous phase had been previously saturated with distilled water of the dispersed phase. The physical properties of the liquids used are shown in Table 1. 2) The effect of flow rate of dispersed phase: the flow rate of dispersed phase was varied in the range prior to jet formation for the cases of cyclohexane and insulating oil, and only a low flow rate was used for the cases of toluene and silicone oils. 3) The effect of nozzle protrusion length: the length was set at 0.1 and $0.4 \mathrm{~cm}$ for the cyclohexane case, and was kept at $0.1 \mathrm{~cm}$ for the others. Experiments could 


\begin{tabular}{|c|c|c|c|c|}
\hline Liquid & $\begin{array}{l}\text { Density } \\
{\left[\mathrm{g} \cdot \mathrm{cm}^{-3}\right]}\end{array}$ & $\begin{array}{c}\text { Interfacial } \\
\text { tension } \\
{\left[\mathrm{dyn}^{\circ} \mathrm{cm}^{-1}\right]}\end{array}$ & $\begin{array}{c}\text { Viscosity } \\
{\left[\mathrm{g} \cdot \mathrm{cm}^{-1} \cdot \mathrm{s}^{-1}\right]}\end{array}$ & $\begin{array}{l}\text { Dielectric } \\
\text { constant } \\
{[-]}\end{array}$ \\
\hline water $^{10)}$ & 0.998 & - & 0.0100 & 80.20 \\
\hline cyclohexane* & 0.770 & 48.0 & 0.0097 & 2.10 \\
\hline toluene* & 0.867 & 34.0 & 0.0060 & 2.38 \\
\hline insulating oil* & 0.872 & 44.6 & 0.105 & 2.23 \\
\hline silicone oil-A* & 0.820 & 40.6 & 0.0088 & 2.29 \\
\hline silicone oil-B* & 0.936 & 37.6 & 0.098 & 2.63 \\
\hline silicone oil-C* & 0.965 & 37.6 & 1.08 & 2.71 \\
\hline
\end{tabular}

not be made for the condition of non-protrusion, because the electrode was wetted frequently by the drop liquid during formation.

\section{Results and Discussion}

\section{1 Uncharged drop formation}

Experimental volumes of uncharged water drops formed in cyclohexane, which were obtained without applying a voltage difference between electrodes, are shown in Fig. 2 as a function of dispersed-phase flow rate. The drop volumes increase slightly with increasing flow rate at first, because of the increase of volumetric flow into the pendant drop during the necking process. When the flow rate is increased further, inertial force due to the dispersed phase flowing into the pendant drop increases and the volumes decrease gradually. The lines in Fig. 2 show the values calculated by Eq. (1) for different $K$-values. The dotted line for $K=0$, which corresponds to drop formation without the necking process, gives volumes smaller than the experimental ones. The chain line indicates Scheele and Meister's prediction for $K=4.5$, which was determined by fitting their experimental data for twelve systems, but it gives much larger volumes than the present experimental ones. This may be due to the difference of experimental methods. That is, in the present work water drops were formed in an organic continuous phase with wetting of the nozzle tip by the dispersed phase liquid, while in Scheele and Meister's experiment organic drops were formed in water continuous phase without wetting of the nozzle tip. To evaluate the effect of the electric field on drop formation, a good correlation for the uncharged drop volumes is required and the $K$-value was newly determined to be 1.15 as a reasonable value (solid line in Fig. 2) for the system of water drop in cyclohexane by fitting the experimental data.

For the system of water drop in insulating oil, the relationship between the experimental $v-Q_{\mathrm{n}}$ values and the values calculated by using the $K$-values of 0 and 4.5 showed tendencies similar to those of water drop in cyclohexane system. The $K$-value was evaluated

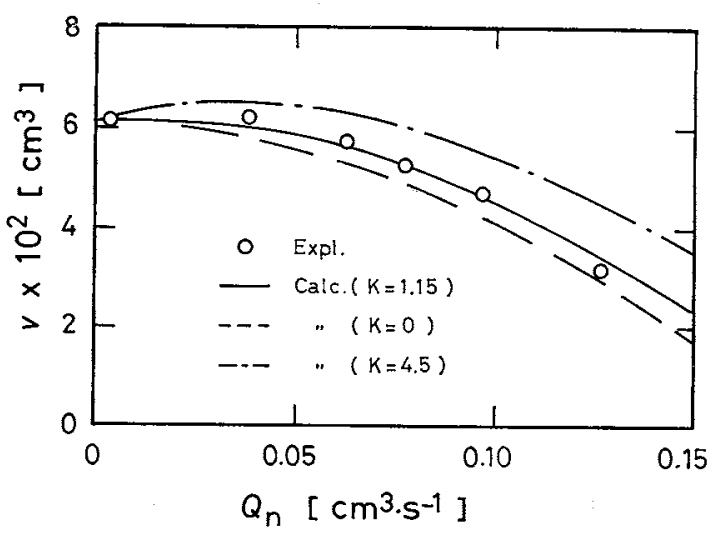

Fig. 2 Dependence of drop volume on dispersedphase flow rate for the system of water drop in cyclohexane $\left(D_{\mathrm{n}}=0.1 \mathrm{~cm}, E_{0}=0 \mathrm{kV} \cdot \mathrm{cm}^{-1}\right)$

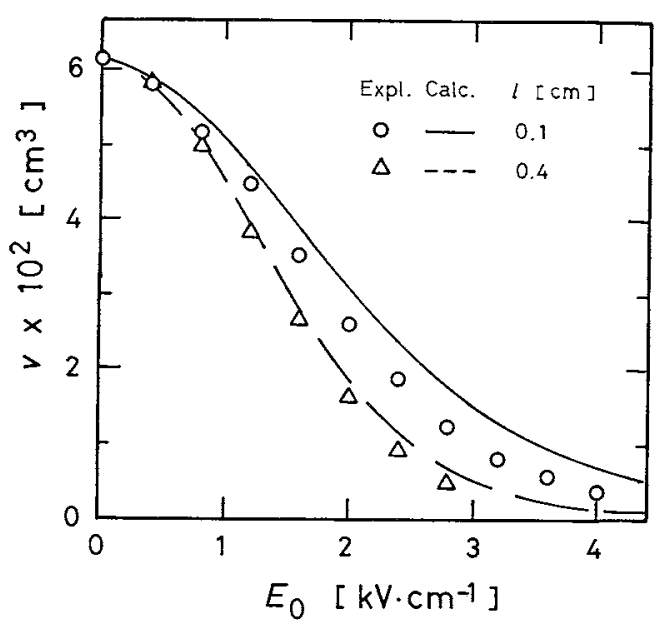

Fig. 3 Drop volume vs. electric field strength for the system of water drop in cyclohexane $\left(D_{\mathrm{n}}=\right.$ $0.1 \mathrm{~cm}, Q_{\mathrm{n}}=0.00322 \mathrm{~cm}^{3} \cdot \mathrm{s}^{-1}$ )

to be 1.84 for the case of insulating oil. The following analysis for charged drops was made by using the value obtained in the experiments for each system.

\section{2 Charged drop formation at low flow rate of dis- persed phase}

Figure 3 shows typical experimental volumes of charged drops formed at a low flow rate of dispersed phase, where the inertial and drag forces and the necking process are negligible. In the figure the drop volumes are plotted against electric field strength (=applied potential/electrode gap) with protrusion length of the nozzle as a parameter. It shows that the drop volume decreases remarkably with increase of electric field strength and also with increasing protrusion length of the nozzle. This fact shows that the specific area of the drop, which is one of the important factors in the operation of liquid-liquid extraction, is changed effectively by protrusion length as well as by field strength. Each line in the figure shows the value calculated by Eq. (2) and is in good 


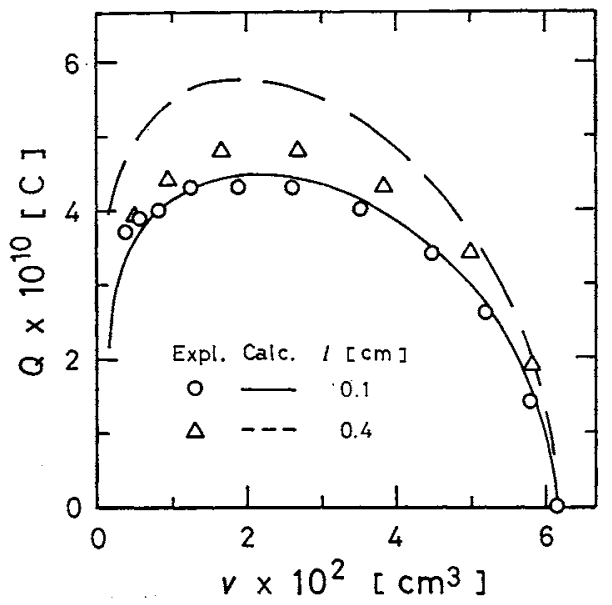

Fig. 4 Drop charge vs. drop volume for the system of water drop in cyclohexane $\left(D_{\mathrm{n}}=0.1 \mathrm{~cm}, Q_{\mathrm{n}}=\right.$ $0.00322 \mathrm{~cm}^{3} \cdot \mathrm{s}^{-1}$ )

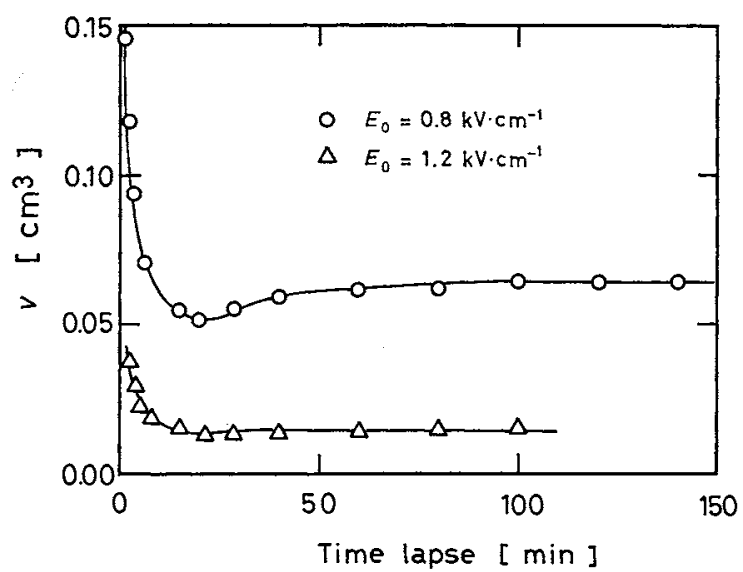

Fig. 5 Effect of time lapse after applying electric field on drop volume for the system of water drop in silicone oil-C $\left(D_{\mathrm{n}}=0.1 \mathrm{~cm}, l=0.1 \mathrm{~cm}, Q_{\mathrm{n}}=6 \times\right.$ $10^{-3} \mathrm{~cm}^{3} \cdot \mathrm{s}^{-1}$ )

agreement with the experimental data. Similar results were obtained for the systems of toluene, insulating oil, silicone oil-A and silicone oil-B as the continuous phase, but for the cases of silicone oil-A and silicone oil-B the agreement is not so good as for the other systems. In our previous study of drop formation in the atmosphere ${ }^{13}$, the experimental drop volumes were always smaller than the ones calculated theoretically. A possible reason why the experimental results showed good agreement with the calculated ones is the small density difference between the water drop and the continuous liquid phase in the present study. That is, because the density differences of liquid-liquid systems used in the present experiment were far smaller than those of gas-liquid systems, the pendant drop would become more like a sphere. Therefore, it is considered that the experimental drop volumes approach the theoretical ones in which the drop is assumed to be spherical.

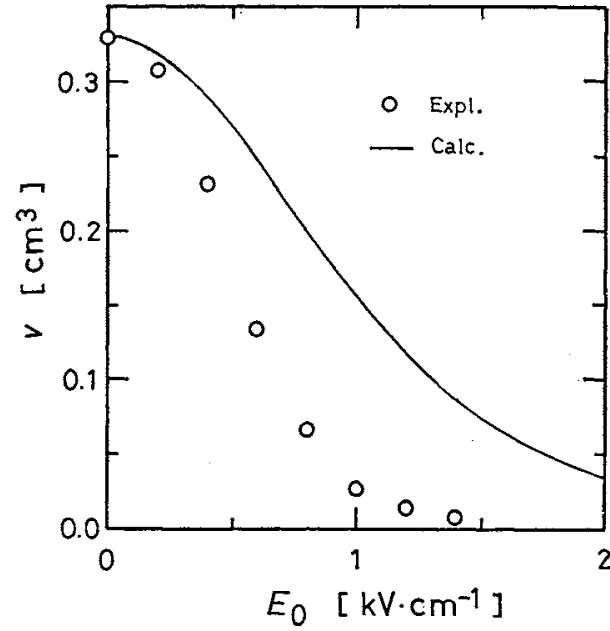

Fig. 6 Drop volume vs. electric field strength for the system of water drop in silicone oil-C $\left(D_{\mathrm{n}}=0.1\right.$ $\left.\mathrm{cm}, l=0.1 \mathrm{~cm}, Q_{\mathrm{n}}=6 \times 10^{-3} \mathrm{~cm}^{3} \cdot \mathrm{s}^{-1}\right)$

The relation between drop charge and drop volume for the system of water drop in cyclohexane is shown in Fig. 4. The experimental data for the case of short protrusion length of nozzle $(0.1 \mathrm{~cm})$ are in good agreement with the theoretical values (solid line) which are calculated by Eq. (4). Similar results were obtained for the systems of toluene, insulating oil, silicone oil-A and silicone oil-B as the continuous phase. When the protrusion length is large $(0.4 \mathrm{~cm})$, however, the theoretical values (the dotted line) are larger than the experimental ones. The tendency of these results was also obtained for gas-liquid systems ${ }^{13}$. The discrepancy between calculated and experimental values in the case of long protrusion length of the nozzle is due to the neglect of the charge on the nozzle in the model as discussed in the previous paper ${ }^{13)}$.

When silicone oil-C of high liquid viscosity was used as the continuous phase, the behavior of drop formation was significantly different from the cases for the other systems. It was found that drop volumes varied remarkably with the lapse of time after applying the electric field. A typical example of time-dependence of drop volume is shown in Fig. 5. Just after applying the electric field, drop volumes rapidly begin to decrease at first and then increase gradually, finally reaching a constant value after about two hours. However, the final volumes, shown in Fig. 6, were always reproduceable for each electric field strength. The experimental drop volumes are much smaller than the theoretical ones calculated by Eq. (2). Though the cause of the time-dependency and the discrepancy of drop volumes is not clear, the following reason is considered: When a voltage difference is applied between parallel-plate electrodes immersed in a stationary liquid without drop formation, a very small current flows between the electrodes 


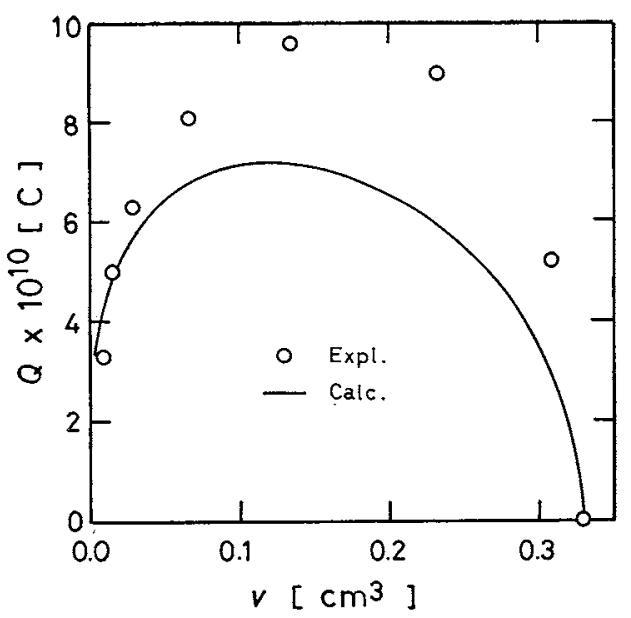

Fig. 7 Drop charge vs. drop volume for the system of water drop in silicone oil-C $\left(D_{\mathrm{n}}=0.1 \mathrm{~cm}, l=0.1\right.$ $\left.\mathrm{cm}, Q_{\mathrm{n}}=6 \times 10^{-3} \mathrm{~cm}^{3} \cdot \mathrm{s}^{-1}\right)$

even if the liquid is a so-called insulator, and a space charge accumulates in the liquid with the lapse of $t^{t i m e} e^{2,3}$. Because the space charge concentrates near surfaces of the electrodes, the potential profile in the electric field is distorted to non-linear form and the field strength near the electrodes is enhanced. The time-dependency of the drop volume and the discrepancy between theoretical and experimental volumes for the system of water drop in silicone oil-C may be due to the enhanced field strength around the drop by accumulation of the space charge. The accumulation will progress with the lapse of the time. That is, the charge carriers and the liquid around the drop as a electrode may not be carried away so rapidly with the detached drops because of high viscosity of the continuous phase. On the contrary, for the cases of cyclohexane, toluene and insulating oil and also for the cases of silicone oils-A and -B, no timedependency or discrepancy was found. It seems that the space charge has no influence on drop formation in low-viscosity liquids where the formation of a constant frequency produces a disturbance of its liquid surroundings.

Experimental electric charges of water drops formed in silicone oil-C are shown in Fig. 7. The experimental values are not in good agreement with the theoretical ones calculated by Eq. (4). This discrepancy is caused by the space charge.

\section{3 Charged drop formation at high flow rate of dis- persed phase}

A typical result of the volumes of charged water drops formed at a high flow rate of dispersed phase in insulating oil as the continuous phase is shown in Fig. 8, together with that at a low flow rate. The drop volumes shown by triangle symbol for the high flow rate decrease with increasing electric field strength in the same manner as those shown by circle symbol

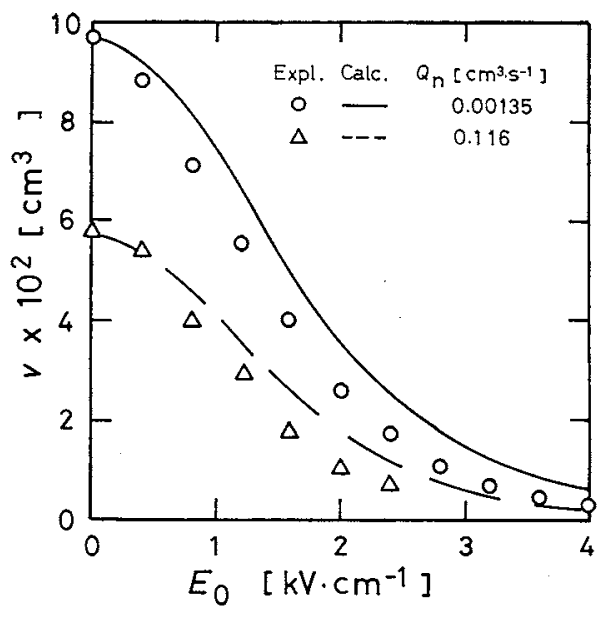

Fig. 8 Drop volume vs. electric field strength for the system of water drop in insulating oil $\left(D_{\mathrm{n}}=\mathbf{0 . 1}\right.$ $\mathrm{cm}, l=0.1 \mathrm{~cm}$ )

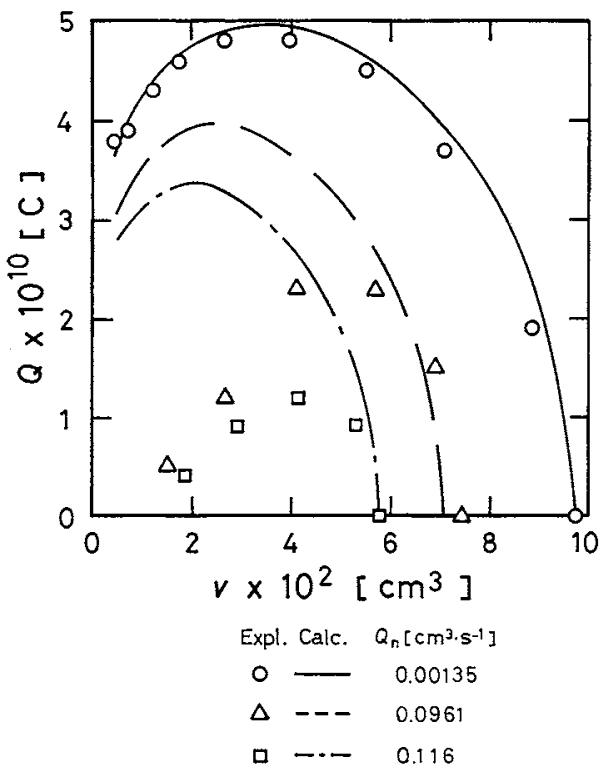

Fig. 9 Drop charge vs. drop volume for the system of water drop in insulating oil $\left(D_{\mathrm{n}}=0.1 \mathrm{~cm}, l=0.1 \mathrm{~cm}\right)$

for the low flow rate. Each line calculated by Eq. (2) shows good agreement with the experimental data. Similar results were obtained for other flow rates and also for the case of cyclohexane. It is concluded that the volumes of single charged drops formed at high flow rates of dispersed phase can be predicted satisfactorily by a semi-empirical equation of Scheele and Meister type in which the electrostatic force given by Eq. (3) is incorporated.

In Fig. 9, experimental drop charges for the system of water drop in insulating oil are plotted against the volumes with a parameter of the flow rate. The charges shown by triangle and square symbols, which increase at first from zero value with increasing electric field strength (though the strength is not expressed directly in the figure), pass through respective maxi- 


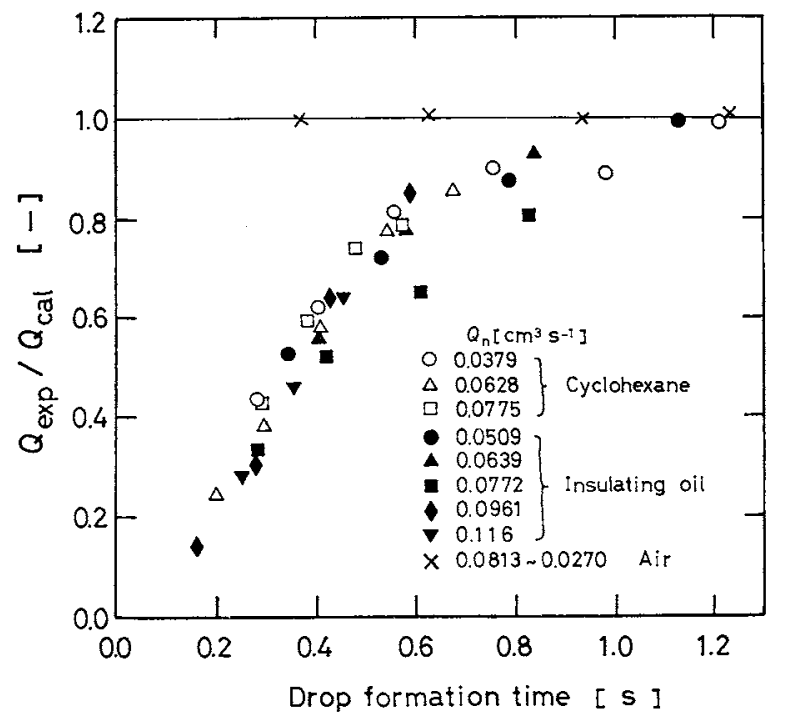

Fig. 10 Relations between drop formation time and drop charge

mum values and then decrease rapidly. As shown in the figure, although the experimental charges for the low flow rate agree well with the calculated ones, the experimental charges for the high flow rates are extremely smaller than the calculated ones. Figure 10 shows the ratio of calculated and experimental drop charges against drop formation time for the systems of water drop in cyclohexane and insulating oil at nozzle protrusion length of $0.1 \mathrm{~cm}$. While there are no systematic differences between the liquids used as the continuous phase, the dependency of $Q_{\mathrm{exp}} / Q_{\mathrm{ca} 1^{-}}$ value on formation time is evident. That is, the values decrease significantly with decreasing formation time, though the calculated and experimental values agree well at low flow rates (in Figs. 4 and 9), i.e. large formation time. For the formation of charged drops in the atmosphere no reduction of the values due to the decrease of formation time was found under $0.4 \mathrm{sec}$. The dependency of $Q_{\mathrm{exp}} / Q_{\mathrm{cal}}$-value on formation time may be based on the manner of charge-accumulation at the liquid-liquid interface of the pendant drop, but further discussion is not possible from these results alone.

\section{Conclusion}

Experimental studies of water-drop formation at an electrified nozzle protruding from one of a pair of parallel-plate electrodes were made in several dielectric liquids at various flow rates of dispersed phase. The experimental results of drop volume and drop charge were studied by comparing them with the values predicted by the theoretical equations presented.

The conclusions of the present work are summarized as follows.

1) Equation (2) can predict satisfactorily the ex- perimental drop volume formed in a continuous phase having viscosity up to ten centipoises over the range of dispersed-phase flow rates prior to jet formation.

2) The length of the nozzle extended from an electrode is one of the important factors which control the drop volume in operation of electrostatic liquidliquid extraction.

3) For systems of water drops in a liquid having viscosity up to ten centipoises, experimental drop charges were in good agreement with the theoretical ones at low flow rates of dispersed phase. However, they were much smaller than the predicted ones at high flow rates of dispersed phase.

4) For the system of silicone oil-C of high viscosity as continous phase, the drop volume depended remarkably on the time lapse after applying the electric field, and experimental drop volumes were much smaller than the theoretical ones.

\section{Acknowledgment}

The authors would like to thank Messrs. T. Kubo, K. Morinaga and Miss R. Ohashi for preparing many fundamental techniques and valuable information of this research. A special note of thanks is extended to the late Mr. T. Katto (1977) for his extraordinary efforts in the early work. Also, the authors are grateful to Mr. T. Nomura for his assistance in the experimental work. This work was partially supported by a Grantin-Aid for Developmental Scientific Research from The Ministry of Education, Science and Culture, Japan.

\section{Nomenclature}

$D_{\mathrm{n}} \quad=$ outer diameter of a nozzle $\quad[\mathrm{m}]$

$D_{\mathrm{n}, \mathrm{in}}=$ inner diameter of a nozzle [m]

$d_{\mathrm{e}} \quad=$ equivalent spherical diameter of a drop [m]

$E_{0} \quad=$ uniform electric field strength $\quad\left[\mathrm{V} \cdot \mathrm{m}^{-1}\right]$

$F_{\mathrm{e}} \quad=$ electrostatic force $[\mathrm{N}]$

$K \quad=$ experimental constant in Eqs. (1) and (2) [-]

$l \quad=$ nozzle length extended from electrode [m]

$Q \quad=$ electric charge of a drop [C]

$Q_{\mathrm{n}} \quad=$ flow rate of dispersed phase through nozzle

$\left[\mathrm{m}^{3} \cdot \mathrm{s}^{-1}\right]$

$r \quad=$ radius of a pendant drop $\quad$ [m]

$u_{\mathrm{n}} \quad=$ dispersed-phase velocity through nozzle

$v \quad=$ volume of a drop $\quad\left[\mathrm{m}^{3}\right]$

$\alpha \quad=(l+r) / r \quad[-]$

$\beta \quad=$ force coefficient, defined by Eq. (3) [-]

$\gamma \quad=$ charge coefficient, defined by Eq. (4) [-]

$\varepsilon \quad=$ permittivity of continuous phase $\quad\left[\mathrm{F} \cdot \mathrm{m}^{-1}\right]$

$\mu_{\mathrm{s}} \quad=$ viscosity of continuous phase $\quad\left[\mathrm{kg} \cdot \mathrm{m}^{-1} \cdot \mathrm{s}^{-1}\right]$

$\rho_{\mathrm{d}} \quad=$ density of dispersed phase $\quad\left[\mathrm{kg} \cdot \mathrm{m}^{-3}\right]$

So $\quad=$ density difference $\quad\left[\mathrm{kg} \cdot \mathrm{m}^{-3}\right]$

$\sigma \quad=$ interfacial tension $\quad\left[\mathrm{N} \cdot \mathrm{m}^{-1}\right]$

$\phi \quad=$ Harkins correction factor $\quad[-]$

\section{Literature Cited}

1) Bailes, P. J. and J. D. Thornton: Proc. Inter. Solvent Extraction Conf., p. 1431, London (1971). 
2) Forster, E. O.: J. Chem. Phys., 37, 1021 (1962), 40, 86, 91 (1964).

3) Forster, E. O.: IEEE Trans., EI-2, 101 (1967).

4) Harker, J. H. and J. Ahmadzadeh: Int. J. Heat Mass Transfer, 17, 1219 (1974).

5) Hendricks, C. D., R. S. Carson, J. J. Hogan and J. M. Schneider: AIAA J., 2, 733 (1964).

6) Kumar, R.: Chem. Eng. Sci., 26, 177 (1971).

7) Mori, Y., K. Hijikata and T. Nagasaki: Trans. Japan Soc. Mech. Engrs. (in Japanese), B-47, 1881 (1981).

8) Ogata, S., T. Kawashima, O. Nakaya and H. Shinohara: J. Chem. Eng. Japan, 9, 440 (1976).

9) Rao, E. V. L. N., R. Kumar and N. R. Kuloor: Chem.
Eng. Sci., 21, 867 (1966).

10) Riddick, J. A. and W. B. Bunger: "Organic Solvents", 3rd ed., John Wiley \& Sons, Inc., New York (1970).

11) Scheele, G. F. and B. J. Meister: AIChE J., 14, 9 (1968).

12) Stewart, G. and J. D. Thornton: Instn. Chem. Engrs. Symp. Ser., 26, 29, 37 (1967).

13) Takamatsu, T., Y. Hashimoto, M. Yamaguchi and T. Katayama: J. Chem. Eng. Japan, 14, 178 (1981).

14) Thornton, J. D.: Birmingham Univ. Chem. Engr, 27, 6 (1976).

15) Vonnegut, B. and R. L. Neubauer: J. Colloid Sci., 7, 616 (1952).

\title{
EFFECT OF NATURAL CONVECTION ON LAMINAR FLOW HEAT TRANSFER IN HORIZONTAL CIRCULAR TUBES
}

\author{
Kunio KATO, ETSUO WATANABE, \\ TAKASHI OGURA AND TAMOTSU HANZAWA \\ Department of Chemical Engineering, Gunma University, Kiryu 376
}

\begin{abstract}
The temperature distribution was measured in a horizontal circular tube with a high uniform wall temperature in the case where gas flowed through the tube in the laminar flow range. The temperature in the upper portion of the cross section was higher than that in the lower portion. The isothermal lines estimated from the measured temperature distribution in the cross section were symmetrical to the vertical centerline of the cross section.

The heat transfer coefficient from the tube wall is affected by tube diameter, distance from tube inlet, wall temperature and Reynolds number. The empirical equation for the heat transfer coefficient from the tube wall is obtained.

To analyze the heat transfer in the tube the fundamental equations are derived under suitable assumptions based upon observation of the flow pattern in the tube and are solved numerically. The calculated temperature distribution and the calculated heat transfer coefficient from the tube wall agree approximately with the experimental findings.
\end{abstract}

\section{Introduction}

The laminar convective heat transfer in a horizontal circular tube has been investigated theoretically and experimentally by many investigators after Graetz's' study. When the fluid, the density of which is strongly affected by temperature, flows through the heated tube, a following secondary flow is generated in the tube. The density near the inside wall of the heated tube decreases, hence fluid near the wall moves upward along the wall surface. Simultaneously, fluid near the center of the cross section moves downward to the bottom. This secondary

Received January 9, 1982. Correspondence cencerning this article should be addressed to $\mathbf{K}$. Kato. E. Watanabe is at Fujitsu Co., Ltd., and T. Ogura is at Chu-Ito Co., Ltd. flow is combined with the axial forced convective flow; consequently, a pair of symmetrical spiral flows is generated in the tube. In this case, the heat transfer coefficient from the tube wall becomes larger than that estimated from Poiseuille flow.

To analyse the heat transfer in the tube, the following two cases are considered: uniform heat flux and uniform wall temperature. Morcos et al..$^{9}$, Hussain and $\mathrm{McComas}^{5)}$, Lichtarowicz' ${ }^{7}$, Mori et al. ${ }^{10)}$ and Shannon Depew ${ }^{13}$ ) obtained the heat transfer coefficient from tube wall under the boundary condition of uniform heat flux.

On the other hand, Sieder and Tate ${ }^{14}$, Brown and Thomas $^{11}$, and Depew and August ${ }^{21}$ obtained the heat transfer coefficient at uniform wall temperature. 